\title{
Intensidad de dosis relativa en pacientes con cáncer tratados con quimioterapia
}

Relative dose intensity (RDI) in patients with cancer treated with chemotherapy

- Pedro Ramos, MD, MSC

Medicina interna, oncología, epidemiología. Clínica Oncocare.

L as intensidades de dosis relativas (IDR) en pacientes con cáncer en tratamiento quimioterápico idealmente deben ser del 100\%; la IDR mayor del $85 \%$ es aceptable y no afectaría los desenlaces duros, como supervivencia global y libre de enfermedad.

En diferentes estudios farmacocinéticos y farmacodinámicos, se ha observado un aumento lineal de la citotoxicidad en función de la dosis administrada, a la vez que disminuye la probabilidad de aparición de tumores resistentes ${ }^{1}$.

Un parámetro considerado de utilidad en la predicción de la efectividad en un tratamiento citostático es la intensidad de dosis (ID $)^{1-3}$ tanto en cáncer de mama como en otro tipo de tumores ${ }^{4-8}$. La ID se define como la cantidad de citostático administrado por superficie corporal (SC) y por unidad de tiempo $\left(\mathrm{mg} / \mathrm{m}^{2} / \text { semana }\right)^{4,8}$.

Algunos estudios indican que obtener una intensidad de dosis relativa igual o superior al $85 \%$ de la programada es un factor predictivo positivo para la supervivencia global y supervivencia libre de enfermedad tanto en quimioterapia adyuvante como en el tratamiento de cáncer de mama metastásico ${ }^{4,9,10}$.

Por el contrario, la disminución en la IDR por debajo del $85 \%$ de la programada, independientemente de la causa, puede considerarse como un factor predictivo negativo clínicamente relevante en la evolución de la enfermedad ${ }^{11-13}$. En este sentido, se estima que más del $60 \%$ de las pacientes que reciben quimioterapia como tratamiento adyuvante para el cáncer de mama experimentan reducciones en la ID programada. Concretamente entre un $21 \%$ y un $30 \%$ de las pacientes recibe menos del $85 \%$ en algunos de los protocolos más frecuentes ${ }^{2,12}$.

Entre las causas, la neutropenia es uno de los principales efectos adversos responsables de la reducción en la IDR, apareciendo en un $29 \%$ de las pacientes bajo tratamiento quimioterápico adyuvante. El 11\% de las pacientes que padecen neutropenia reciben menos del $85 \%$ de la IDR programada.

El retraso en la administración del ciclo quimioterápico igual o superior a siete días es el procedimiento más frecuentemente utilizado para minimizar la toxicidad; otra opción es la reducción de dosis del citostático, que también tiene repercusión sobre la IDR final'12-14.

El aumento progresivo de la edad de las pacientes afecta la IDR recibida por las toxicidades mayores que obligan a disminuir dosis o prolongar los intervalos de tratamiento, situación que puede mitigarse mejorando las terapias de soporte. 
La valoración geriátrica integral (VGI) es un proceso diagnóstico global, diseñado para identificar los problemas clínicos, funcionales, psíquicos y sociales que presenta el paciente geriátrico, de uso rutinario en geriatría, pero no es usada por los oncólogos en la práctica clínica rutinaria.

Debido a que la VGI conlleva una evaluación que abarca una cantidad de tiempo prolongada, se ha buscado la aplicación de una herramienta que incorpore factores geriátricos y oncológicos de vulnerabilidad para desarrollar toxicidad relacionada con el tratamiento con quimioterapia que sea más práctica. Este instrumento podría ayudar al médico y al paciente a tomar decisiones para el tratamiento con base en el peso del riesgo y beneficio de este a fin de evitar el riesgo de desarrollo de la toxicidad por quimioterapia. El modelo consiste en 11 variables prequimioterapia que incluyen preguntas de evaluación geriátrica, valores de laboratorio, características del tumor, tratamiento planeado y edad ${ }^{15}$.

Otro factor es el número de combinaciones citostáticas recibidas previamente por la paciente que afectan su reserva medular.

El concepto de intensidad de dosis, aplicado a los tratamientos quimioterápicos que reciben en la práctica diaria nuestros pacientes, es un indicador específico que nos permitirá cuantificar la calidad de atención en salud y valorar la necesidad de posibles medidas concomitantes y de planificación en los esquemas quimioterapéuticos. La finalidad sería afectar en la menor medida posible la intensidad de dosis recibida, teniendo en cuenta así los diferentes factores que pueden influir en ella, como la reducción de dosis de la quimioterapia por toxicidad, factor que más contribuye en la reducción de la IDR a nivel internacional; en nuestro medio es más frecuente la prolongación en el tiempo de aplicación, más por temas administrativos de autorización de las entidades promotoras de salud (EPS). La supervisión de la dosis que realmente se les administra a los pacientes, junto con las medidas concomitantes para favorecer su administración, es una tarea multidisciplinaria, en la cual es clave concientizar a las EPS de esta problemática, que los organismos de control sean más vigilantes de esta situación y se tomen los correctivos de forma integral, lo cual tendrá como objetivo principal la mejora de la efectividad del tratamiento quimioterápico del cáncer, impactando los desenlaces importantes, como la supervivencia global y libre de enfermedad ${ }^{16,17}$.

En el estudio de Sánchez y colaboradores publicado en esta edición, se muestra el impacto en nuestro medio de la disminución de la intensidad de dosis en pacientes ancianas; se evidencia una alta frecuencia de pacientes que reciben intensidad de dosis subóptima menor al $85 \%$ y unas respuestas patológicas completas a la neoadyuvancia en cáncer de mama menor que la observada en estudios internacionales y locales. Un 38,8\% de las pacientes tuvieron una intensidad de dosis $\geq 85 \%$. La tasa de respuesta patológica completa global fue de un $16,39 \%$. La respuesta patológica completa en pacientes con sobreexpresión del HER2 fue del 23,5\%, en triple negativo fue del $23 \%$ y luminal, del $11,36 \%$. Se requieren seguimientos más prolongados para ver el impacto en la supervivencia global y libre de enfermedad, ya que las respuestas patológicas son un predictor de la supervivencia.

\section{Referencias}

1. Budman DR, Berry DA, Cirrincione CT, Henderson IC, Wood WC, Weiss RB, et al. Dose and dose intensity as determinants of outcome in the adjuvant treatment of breast cancer. The Cancer and Leukemia Group B. J Natl Cancer Inst. 1998;90(16):1205-11.

2. Chang J. Chemotherapy dose reduction and delay in clinical practice. Evaluating the risk to patient outcome in adjuvant chemotherapy for breast cancer. Eur J Cancer. 2000;36 Suppl 1:S11-4.

3. Wood WC, Budman DR, Korzum AH, Cooper MR, Younger J, Hart RD, et al. Dose and dose intensity of adjuvant chemotherapy for stage II, node-positive breast carcinoma. N Engl J Med. 1994;330(18):1253-9.
4. Hryniuk $W$, Bush $H$. The importance of dose intensity in chemotherapy of metastatic breast cancer. J Clin Oncol. 1984;2(11):1281-8.

5. Hryniuk W, Frei E 3rd, Wright FA. A single scale for comparing dose-intensity of all chemotherapy regimens in breast cancer: summation dose-intensity. J Clin Oncol. 1998;16(9):3137-47.

6. Hryniuk W, Levine MN. Analysis of dose intensity for adjuvant chemotherapy trials in stage II breast cancer. J Clin Oncol. 1986;4(8):1162-70.

7. Berg J. Dose intensity in patients with metastatic breast cancer--time for novel thoughts? Ann Oncol. 1997;8(2):109-10. 
8. Hryniuk W, Goodyear M. The calculation or received dose intensity. J Clin Oncol. 1990;8:1935-37.

9. Hamilton A, Hortobagyi G. Chemotherapy: what progress in the last 5 years? J Clin Oncol. 2005;23(8):1760-75.

10. Bonadonna $G$, Valagussa $P$, Moliterni $A$, Zambetti $M$, Brambilla C. Adjuvant cyclophosphamide, methotrexate, and fluorouracil in node-positive breast cancer: the results of 20 years of followup. N Engl J Med. 1995;332(14):901-6.

11. Berry DA, Budman DR. Dose and dose intensity as determinants of outcome in the adjuvant treatment of breast cancer. J Nat Cancer Inst. 1999;91(16):1425A-1425.

12. Shayne M, Crawford J, Dale DC, CulaKova E, Lyman GH; ANC Study Group. Predictors of reduced dose intensity in patients with early-stage breast cancer receiving adjuvant chemotherapy. Breast Cancer Res Treat. 2006;100(3):255-62.
13. Lyman GH, Dale DC, Crawford J. Incidence and predictors of low dose-intensity in adjuvant breast cancer chemotherapy: a nationwide study of community practices. J Clin Oncol. 2003;21(24):4524-31.

14. Piccart MJ, Biganzoli L, Di Leo A. The impact of chemotherapy dose density and dose intensity on breast cancer outcome: what have we learned? Eur J Cancer. 2000;36 Suppl 1:S4-10.

15. Hurria A, Mohile S, Gajra A, Klepin H, Muss H, Chapman A, et al. Validation of a prediction tool for chemotherapy toxicity in older adults with cancer. J Clin Oncol. 2016;34(20):2366-71.

16. Pérez J, Cholvi $M$, Almela $M$, Borrás $C$, Almenar $D$, Jiménez $N$. Análisis de la intensidad de dosis y la adhesión de los esquemas de quimioterapia adyuvante en pacientes con cáncer de mama. Atención Farmacéutica. 2000;2:211-8.

17. Ziegler J, Citron M. Dose-dense adjuvant chemotherapy for breast cancer. Cancer Nurs. 2006;29(4):266-72. 